\title{
DISTINCT FRONTAL REGIONS FOR PROCESSING SENTENCE SYNTAX AND STORY GRAMMAR
}

\author{
A. Sirigu ${ }^{1}$, L. Cohen ${ }^{2}$, T. Zalla ${ }^{1,3}$, P. Pradat-Diehl ${ }^{3}$, P. Van Eeckhout ${ }^{2}$, J. Grafman ${ }^{4}$ \\ and Y. Agid ${ }^{1}$ \\ ( ${ }^{1}$ Inserm U289; ${ }^{2}$ Service de Neurologie, ${ }^{3}$ Service de Rééducation Neurologique, Hôpital de \\ la Salpêtrière, Paris, France; ${ }^{3}$ CREA, Ecole Polytechnique; ${ }^{4}$ Cognitive Neuroscience \\ Section, NINDS, NIH, Bethesda)
}

\begin{abstract}
Time is a fundamental dimension of cognition. It is expressed in the sequential ordering of individual elements in a wide variety of activities such as language, motor control or in the broader domain of long range goal-directed actions. Several studies have shown the importance of the frontal lobes in sequencing information. The question addressed in this study is whether this brain region hosts a single supramodal sequence processor, or whether separate mechanisms are required for different kinds of temporally organised knowledge structures such as syntax and action knowledge. Here we show that so-called agrammatic patients, with lesions in Broca's area, ordered word groups correctly to form a logical sequence of actions but they were severely impaired when similar word groups had to be ordered as a syntactically well-formed sentence. The opposite performance was observed in patients with dorsolateral prefrontal lesions, that is, while their syntactic processing was intact at the sentence level, they demonstrated a pronounced deficit in producing temporally coherent sequences of actions.

Anatomical reconstruction of lesions from brain scans revealed that the sentence and action grammar deficits involved distinct, non-overlapping sites within the frontal lobes. Finally, in a third group of patients whose lesions encompassed both Broca's area and the prefrontal cortex, the two types of deficits were found. We conclude that sequence processing is specific to knowledge domains and involves different networks within the frontal lobes.
\end{abstract}

Key words: sequence processing, frontal lesion, neuropsychology

\section{INTRODUCTION}

A central issue in cognitive neuroscience is how elements are bound together into temporally ordered hierarchies. Although the frontal lobes have long been thought to play a role in this function, little is known about the way in which sequences are represented in this brain region. As previously suggested for working memory functions (Wilson, O’Scalaidhe and Goldman-Rakic, 1993; Goldman-Rakic, 1997) it is possible to speculate that sequence processing is organised in a modular manner within the frontal cortex. A rationale for a cortical division of labour within frontal areas is suggested by the possibility that different knowledge structures are processed within different temporal windows. For instance, complex goal-directed actions may require specific mechanisms to 
cope with the long-range temporal frame within which they typically unfold, while assembling words to form a sentence takes place in a comparatively much smaller time frame. Another basis for proposing multiple modes of sequence representation is the fact that the nature of the rules which determine temporal order may vary as a function of knowledge domain, e.g. action sequences emphasize natural causality and mean-ends relations, while word order in speech is constrained by the intrinsic structure of language (Chomsky, 1988).

Observations made in patients with focal brain damage appear to support such a hypothesis. The prefrontal cortex has been implicated in the storage and retrieval of knowledge about the sequential aspects of planning; patients with lesions in this region are impaired in planning the temporal order of series of actions both in the laboratory setting and in every-day life activities (Petrides and Milner, 1982; Shallice, 1988; Sirigu, Zalla, Pillon et al., 1995, 1996; Goel and Grafman, 1997). In contrast, patients with lesions involving the left inferior frontal gyrus, or Broca's area, have difficulties with grammatical analysis and word order in spoken and written language (Saffran, Schwartz and Marin, 1980; Caramazza, Berndt, Basili et al., 1981). No studies yet have tried to determine whether impairments in planning action and word sequences can be dissociated.

We examined sequence processing in ten brain-damaged subjects who were assigned to one of three groups. Four aphasic patients had suffered from a left hemisphere infarct involving Broca's area and surrounding regions. These patients presented agrammatic speech, characterised by sparse but informative verbal output, impoverished syntactic structure, reduced function words and grammatical endings. A second group of four patients had lesions located in the left prefrontal cortex and presented deficits in laboratory tasks of planning and in managing everyday life activities. Finally, two further patients had lesions which included Broca's and near rolandic areas and extended into the prefrontal cortex. These patients showed a speech disorder similar to the four Broca's aphasic, but also had impairments in executive functions beyond their language disorders.

\section{MAterials AND Methods}

\section{Subjects}

The first group (Broca) comprised two females and two males (mean age: $52.5 \mathrm{yrs}$, S.D. 4.5; mean education: 17,7 yrs, S.D. 2.1) with vascular lesions in the territory of the left middle cerebral artery (Figure $1 \mathrm{a}$; mean time since lesion $=3.8$ years, range $=1-6$ years). All presented disorders of language production with agrammatic speech, while oral and written language comprehension were better preserved. Despite long and intensive speech therapy, verbal fluency remained largely reduced. The second group (Prefrontal), included two females and two males (mean age: 55.2 yrs, S.D. 6.6; mean education: 16 yrs, S.D. 4.3) with focal damage in the left prefrontal areas (Figure 1b). Three of them suffered from vascular lesions and one sustained a focal trauma. These patients presented impairments in daily-life activities, and two of them also exhibited socially inadapted behaviours, as reported by close relatives. They were all impaired in planning tasks such as the Tower of Hanoi or the Wisconsin Card Sorting Test. One patient in addition presented so-called utilisation behaviour (Lhermitte, Pillon and Serdaru, 1986). No language disorders were observed in this group with the exception of one patient who showed a mild dysarthria. 


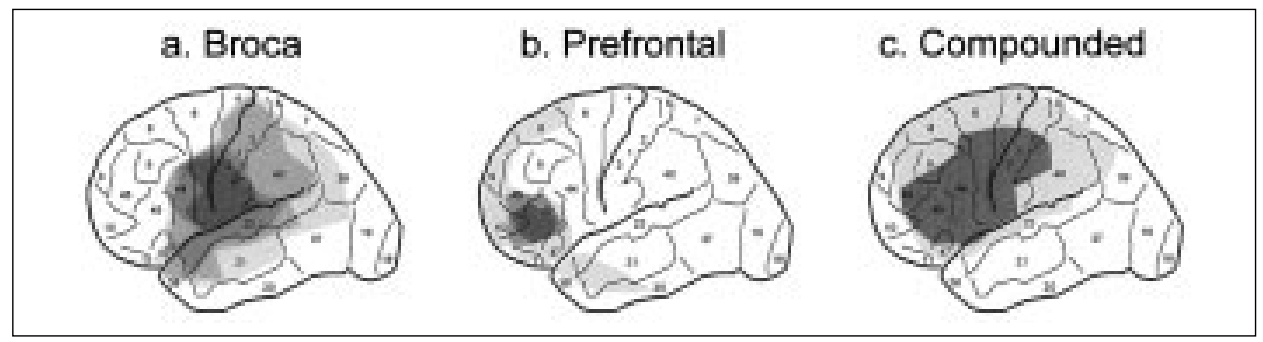

Fig. 1 - Schematic reconstruction of patients' lesions. Data from MRI scans showing areas of intersection of lesions within each patient group. For each reconstruction the lightest area correspond to the lesion of a single patient, the brightest to the lesioned cortical region common to all patients in each group.

The third group (Compounded), was composed of two males (age: 49 yrs and 54 yrs; education: both $18 \mathrm{yrs}$ ) who suffered from vascular left frontal lesions involving areas common to the subjects in the Broca and the Prefrontal groups (Figure 1c). They also showed characteristic neuropsychological deficits of each of these two groups. Five healthy subjects (Ncs) participated in the study as controls (2 males, 3 females, mean age: 51.8, S.D. 4.0; mean education: 10.6, S.D. 4.6).

\section{Procedure}

All subjects were tested on two separate, matched tasks which involved ordering four to five groups of words printed on cards.

\section{Syntax}

In the 'Syntax' task, the goal was to produce a grammatically correct sentence. We used thirty sets of cards, each set forming a complete sentence. Each sentence was split into five segments of one to four words. Individual segments were obtained by splitting the sentence around key grammatical links. In half of the sentences, the substantive of the subject and object were semantically reversible. In the following example: "a lady was / pushed by / a man / while she / crossed the street", 'lady' and 'man' are reversible (although the segments which contain them are themselves non-reversible). In the other half, subject and object were not semantically reversible, for example: "the butcher / sharpens his / knife / and cuts a / thick steak". The presence of semantically non-reversible segments is used to establish the capacity to process word order on the basis of syntactic rules only as opposed to semantic/contextual information. Sentences were matched across the three experimental sets for word frequency, reversibility, syntactic structure and segmentation pattern. For each sentence, there was one single correct ordering of segments, which could be determined only on the basis of morphosynctatic knowledge. This was especially true for semantically reversible sentences, for which strategies such as systematically putting the animate item before the verb, which may be available to some agrammatics, are not operative. One error was scored for each sentence containing at least one incorrectly ordered card, for a maximum of thirthy possible errors for the whole task.

\section{Scripts}

This test was a modified version from the one used in a previous study (Sirigu et al., 1996) and comprised a set of sixty cards with an action written on each one. The goal was to produce a logically consistent short narrative, based on the temporal sequence of actions depicted on the cards, for example: "arrive at the newstand / ask for the paper / take it / pay / leave" or "insert card / pick-up the receiver / dial / waiting for an answer / talk". A stack of twenty cards with a single action written on each one were given to the subjects. The twenty actions described four different unrelated scripts. There were five actions for 
each script, each corresponding to a salient step in the sequence. Each action was described by one to four words. The subject was shown a complete set of twenty cards and requested to sort the cards according to the script the actions belong to and according to their order of execution within each script. They were also shown on a separate sheet the title of the four different scripts which had to be assembled using these cards. The subjects first sorted the cards corresponding to each script, and subsequently ordered each subset of cards according to the chronological order of the actions. Each of the twenty actions could be associated semantically to only one of the four different scripts. This two-step procedure (sorting and ordering) was used to distinguish analysis of a script content from the processing of its temporal sequence. Thus, while in the Syntax task the emphasis was on the grammatical structure of the sentence, the Script task involved the analysis of action grammar. Word frequency, number of words and number of cards per set (i.e. a single sentence or script) were balanced between the Syntax and the Script conditions. A sequence error was scored when the order of at least one action was reversed within each script, for a maximum of four errors per set and a total of twelve errors for the whole task.

\section{RESULTS}

The results were evaluated using analyses of variance and showed that normal subjects made no errors on both tasks. Patients' performance on each task strongly depended on lesion location (Figure 2). Patients with lesions involving Broca's area were severely impaired regarding the number of correctly ordered sentences $(\mathrm{F}\{2,7\}=24.6, \mathrm{p}<.0001)$ compared to those with prefrontal

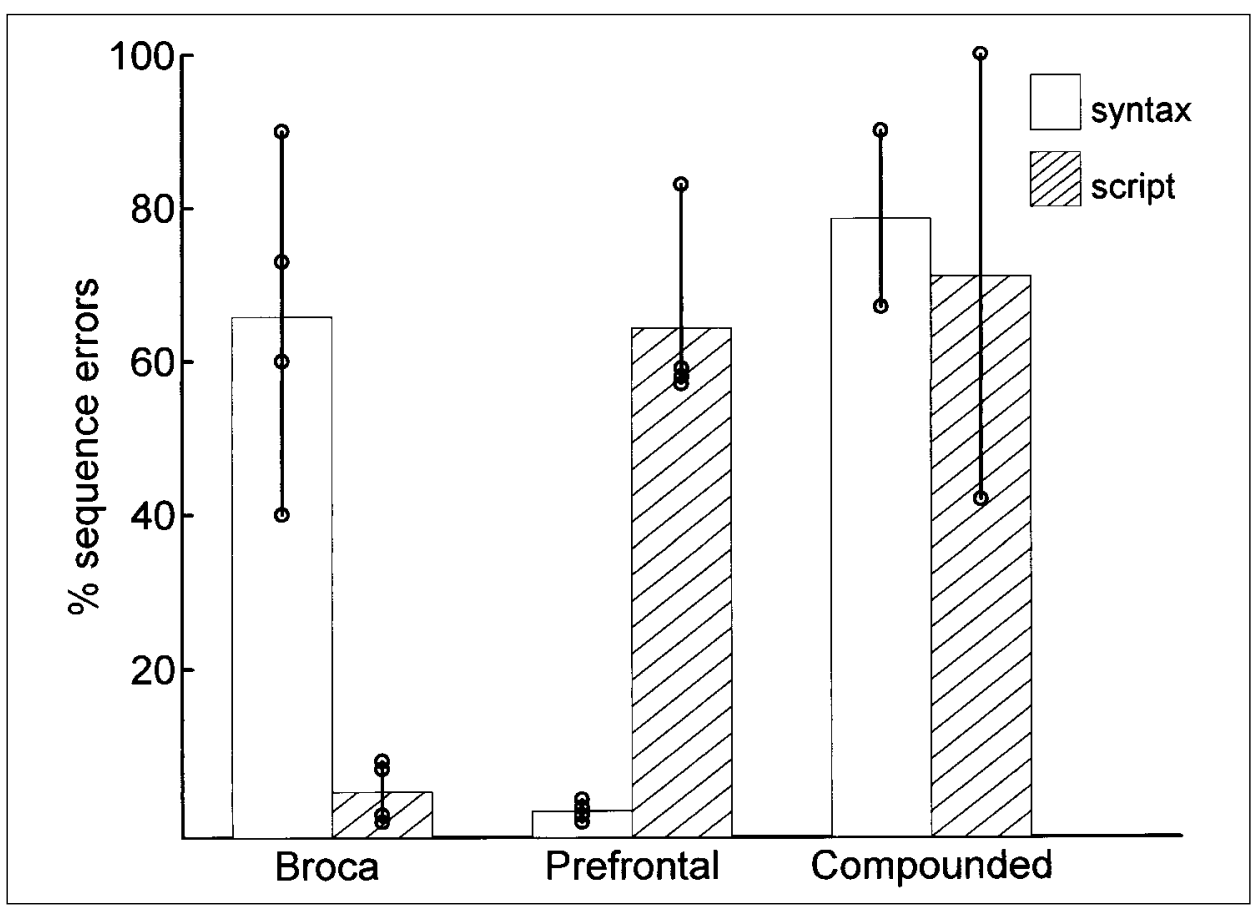

Fig. 2 - Percentage of sequence errors for the three groups of patients and for each task. Dots within the bars represent individual subject's performance. 
lesions, who did not differ from normal subjects. Typical sentences produced by Brocas were: "the husband of / less likeable / my aunt / is much / than my cousin" or "gave a / kiss to / the boy / the lady who / it is". All of these patients, including a highly literate former editor and novelist, produced such incoherent sequences. They spontaneously admitted that something was wrong with their productions but were unable to improve them no matter how long they were allowed to work on the task. Detailed analysis of the errors made by the aphasics showed a higher, though not statistically significant, frequency of errors in reversible than non-reversible sentences (mean $=11$, S.D. 2.1, and mean $=7.2$, S.D. 3.3, respectively, $\mathrm{p}=.07$ ). More importantly, subject/object inversions occurred in about $65 \%$ of the reversible sentences, while such inversions never occurred in non-reversible ones. Thus, the impairment observed in Broca's aphasics pertains specifically to syntax and not to the semantic constraint on word order which define the subject and the object of an action (verb). In contrast, these patients performed like normal subjects on the Script task, correctly sorting and ordering all actions. The opposite pattern was observed in patients with lesions restricted to the prefrontal cortex. These patients made virtually no errors in the Syntax task. In the script task they correctly associated the actions to the proper script theme but they were severely impaired, relative to both normals and Broca's, in ordering the actions within a script in the proper chronological sequence $(\mathrm{F}\{2,7\}=9.7, \mathrm{p}<.01)$. A representative sequence produced by a prefrontal patient is: "tip the usherette / waiting in line / walk into the theatre / take a seat / get a ticket" or "get to the store / pay / order the ice cream / take it / choose the flavour / leave". Such incoherent sequences were observed in all patients of the prefrontal group. Contrary to the aphasic subjects however, they were unaware of the errors and if subsequently probed by the experimenter, they could offer post-hoc justification for their performance. Patients with compounded lesions in Broca's area and the prefrontal cortex were impaired in both tasks relative to normal subjects $(\mathrm{p}<.005)$.

Time to complete each task also depended on lesion location. Normal subjects employed about the same total amount of time to complete the Syntax and Script tasks (mean $=5.6 \mathrm{~m}$, range $=4-7 \mathrm{~m}$, and mean $=6.0 \mathrm{~m}$, range $=4-$ $7 \mathrm{~m}$, respectively). Patients were overall slower than normals, but the time employed varied as a function of task condition. Broca's aphasics were significantly slower in the Syntax than in the Script task (mean $=34 \mathrm{~m}$, range $=$ $19-46 \mathrm{~m}$ and mean $=25 \mathrm{~m}$, range $=12-28$, respectively, $\mathrm{p}<.05)$. Prefrontal patients showed the opposite, though not significant trend (mean $=10 \mathrm{~m}$, range $=9-17 \mathrm{~m}$ for Syntax and mean $=22 \mathrm{~m}$, range $=12-44 \mathrm{~m}$ for Script, ns). Patients with both types of lesions were slow regardless of task condition (mean $=43 \mathrm{~m}$, range $=37-49 \mathrm{~m}$ for Syntax and mean $=32 \mathrm{~m}$, range $=25-40 \mathrm{~m}$ for Script).

\section{DISCUSSION}

In this study we observed a double dissociation of performances on the Syntax and Script tasks. Although in both conditions patients had to order 
words, performance changed depending on the required sequencing principles. Broca's aphasics were impaired when the task stressed syntactic constraints on word order, whereas prefrontals had difficulties when the sequence was related to the pragmatic constraints of action order. Hence, ordering seems not to be dependent on a supramodal processor, but is rather a function of the type of underlying knowledge structure to be processed.

It could be argued that the distinction made between these two types of sequences is really one between semantics and syntax, the results showing that semantics can be processed without syntax and vice-versa. Accordingly, the aphasic patients could have sorted action sequences on the basis of semantic analysis rather than according to specific knowledge of temporal hierarchies. However, if such were the case, the prefrontal patients should also have succeded in the Script task because they did not have any basic semantic impairment. For instance, prefrontal patients as well as patients with lesions in both the prefrontal cortex and Broca's areas were able to correctly assign actions to each script. Furthermore, in a previous study (Sirigu et al., 1995) we showed that prefrontal patients, when asked to evoke a plan of action, e.g. 'prepare a trip' or 'organise your morning routine', generated as many prototypical actions as normals despite the fact that the temporal ordering of the same actions was incorrect. Prototypicality is a parameter which reflects how representative a given action is of its semantic category. This suggests that the ability to process sequences is not simply a by-product of general semantic knowledge but may be a specific component of action knowledge.

Certain neuropsychological deficits observed in patients with frontal lobe lesions have been interpreted in terms of working memory impairments (Freedman and Oscar-Berman, 1981; Verin, Partiot, Pillon et al., 1993). It could be argued that the present result can be explained by a general working memory deficit rather than being a specific impairment of action sequence processing. Such a hypothesis, in the context of the present functional dissociation would have to postulate two different types of working memory impairment, one relative to sentence analysis, the other relative to script level information. This would be consistent with the notion of domain-specific modular working memory systems. However, the tasks used in the present study were designed such as to reduce working memory load since the information chunks that had to be manipulated were written on cards that remained visible throughout the experiment. Therefore, it seems more appropriate to interpret the deficit in terms of the main cognitive operation, namely serial ordering, that had to be performed within each domain: sentence vs. narrative.

The analysis of the cortical areas associated with impaired performance on the Syntax and Script task points to two distinct non-overlapping lesion sites within the frontal cortex (Figure 1). The lesioned area common to all patients impaired in the Syntax but not in the Script task included the left anterior parietal cortex, the left ventral premotor cortex (area 6) and Brodmann's area 44 (Figure 1a). Thus, it seems that, consistent with previous reports of Broca's aphasia (Damasio, 1986), the portion of the left frontal operculum including area 44 and part of area 16 is an important region for processing word sequences at the syntactic level. Patients who were selectively impaired on the Script task had 
more anteriorly located left hemisphere lesions which invariably included area 45 and part of area 46, and which in addition could extend to areas 10, 9, 11 and part of area 8 in the medial frontal gyrus (Figure 1b). None of the patients in this group had lesions in area 44. Finally, patients with deficits in both the Syntax and Script tasks had lesions which included the two identified critical regions within the left frontal lobe (Figure 1c).

The present results support the existence of a subdivision within the human frontal cortex among sequence processes based on two distinctive forms of knowledge representation: sentence syntax and story grammar. This is consistent with the hypothesis that representations within the frontal cortex are categorically organised (Grafman, 1995; O'Scalaidhe, Wilson and GoldmanRakic, 1997). Neuropsychological tasks involving the manipulation of story level knowledge likely tap into the same underlying representations and processes which are used to plan and monitor long range of everyday life behaviour, during which numerous irrelevant events take place between the onset and the completion of a given course of action. A possible neural mechanism for this kind of processing has been identified in experiments showing that prefrontal cortex neurons may be specialised for holding information over a long time frame and across several intervening events as compared to neurons in the temporal cortex which are more sensitive to shorter temporal windows (Miller, Erickson and Desimone, 1996). In contrast, the more posterior frontal cortical regions which are closely tied to motor processing may be better suited for handling the rapid analysis of word order in the context of on-line speech production. It thus seems possible to speculate on the existence of a posterior to anterior gradient of neuronal populations in the frontal cortex being increasingly sensitive to longer temporal windows of information processing, thus subserving the more refined subdivisions of knowledge-linked sequence processing we have identified here.

Acknowledgements. We thanks Dr. B. Pillon for his help during patients' baseline testing. Founded by GIS-CNRS and Fondation pour la Recherche Médicale to AS.

\section{REFERENCES}

Caramazza, A., Berndt, R.S., Basili, A.G., and Koller, J.J. Syntactic processing deficits in aphasia. Cortex, 17: 333-348, 1981.

Chomsky, N. Language and Problems of Knowledge. Cambridge, MA: MIT Press, 1988.

DAmAsio, H. Neuroimaging contributions to the understanding of aphasia. In F. Boller and J. Grafman (Eds.), Handbook of Neuropsychology, vol. 2. Amsterdam: Elsevier, 1989, pp. 3-46.

Freedman, M., and Oscar-Berman, M. Bilateral frontal lobe disease and selective delayed-response deficits in humans. Behavioral Neuroscience, 100: 337-342, 1986.

Goel, V., Grafman, J., TajiK, J., Gana, S., and Danto, D.A. A study of the performance of patients with frontal lobe lesions in a financial planning task. Brain, 1998 (in press).

GoldMAN-RAKIC, P.S. Space and time in the mental universe. Nature, 386: 599-600, 1997.

Grafman, J. Similarities and distinctions among current models of prefrontal cortical functions. Structure and Functions of the Human Prefrontal Cortex, In Grafman, J., Holyoak, K., Boller, F. (Eds.), 337-368. Annals of N.Y. Ac. of Sc., 1995.

Lhermitte, F., Pillon, B., and Serdary, M. Human autonomy and the frontal lobes. I: Imitation and utilisation behavior: A neuropsychological study of 75 patients. Annals of Neurology, 19: 326-334, 1986.

Miller, E.K., Erickson, C.A., and Desimone, R. Neural mechanisms of visual working memory in prefrontal cortex of the macaque. Journal of Neuroscience, 16: 5154-5167, 1996.

O'Scalaidhe, S.P., Wilson, F.A.W., and Goldman-RaKic, P.S. Areal segregation of face-processing 
neurons in prefrontal cortex. Science, 278: 1135-1138, 1997.

Petrides, M., and Milner, B. Deficits on subject-ordered task after frontal and temporal lobe lesions. Neuropsychologia, 20: 249-262, 1982.

Petrides, M., Alivisatos, B., Meyer, E., and Evans, A.C. Functional activation of the human frontal cortex during the performance of verbal working memory tasks. Proceedings of National Academic of Science U.S.A., 90: 878-882, 1993.

SAFFran, E.M., Schwartz, M.F., and Marin, O.S.M. The word order problem in agrammatism. Brain and Language, 10: 249-262, 1980.

Sirigu, A., Zalla, T., Pillon, B., Dubois, B., Grafman, J., and Agid, Y. Selective impairments in managerial knowledge in patients with pre-frontal cortex lesions. Cortex, 31: 301-316, 1995.

Sirigu, A., Zalla, T., Pillon, B., Dubois B., Grafman, J., and Agid, Y. Encoding of sequence and boundaries of script following prefrontal lesions. Cortex, 32: 297-310, 1996.

Verin, M., Partiot, A., Pillon, B., Malapani, C., Agid, Y., and Dubois, B. Delayed response tasks and prefrontal lesions in man: Evidence for self-generated patterns of behavior with poor environmental modulation. Neuropsychologia, 31: 1379-1396, 1993.

Wilson, F.A.W., O'Scalaidhe, S.P., and Goldman-RAKIC, P.S. Dissociation of object and spatial processing domains in primate prefrontal cortex. Science, 260: 1955-1958, 1993.

Dr. Angela Sirigu, CNRS-Institut des Sciences Cognitives, 67, Boulevard Pinel, 69675, Bron, France.

E-mail: sirigu@isc.cnrs.fr

(Received 15 April 1998; accepted 20 May 1998) 